\title{
Root orientation of four sorghum cultivars: application to estimate root length density from root counts in soil profiles
}

\author{
Jean-Louis Chopart ${ }^{1}$, Bassirou Sine ${ }^{2}$, Abdalla Dao $^{2}$ and Bertrand Muller ${ }^{2,3}$ \\ ${ }^{1}$ Annual Cropping Systems Research Unit, CIRAD, 7 ch. de l'IRAT, 97410 St-Pierre, Réunion, France. \\ ${ }^{2}$ Training and research regional center for food security and dry environments (CERAAS), BP 3320, Thiès, Senegal. \\ ${ }^{3}$ Integrative Crop Modeling Research Unit, CIRAD, avenue Agropolis, 34398 Montpellier CEDEX 5, France \\ Corresponding author: J.L. Chopart, E-mail: chopart@cirad.fr, Phone: + 262 499262, Fax: + 262499295
}

Received on July 9, 2008; Accepted on October 3, 2008

\begin{abstract}
The root length density $(R L D)$ distribution in the soil is a key factor for water and nutrient uptake but its direct assessment is not easy. A field method is presented here to assess $R L D$ of sorghum (Sorghum bicolor L. Moench) from the root intersection density $(R I D)$ and taking root orientations into account. The method was developed with four varieties cropped in 2006 in Bambey (Senegal) on a sandy soil, and validated on the basis of independent data obtained on two other varieties cropped in 2008 in Thiès in Senegal on a sandy loam soil. Sets of $1 \times 10^{-3} \mathrm{~m}^{3}$ cubic undisturbed soil samples were collected at different positions and at three different dates for the four varieties during the first experiment. RIDs of thick and fine roots were assessed on the sides of the soil cubes and $R L D s$ were measured in the volumes. Fine roots appeared to be isotropic when thick roots were horizontal near the surface and gradually became vertical in deeper horizons. RLD was tenfold lower for thick roots than for fine ones. This led to the development of a model to determine RLD from RID. The relationship for all roots was modeled as $R L D=R I D \times C O$, where the root orientation coefficient $(C O)$ is equal to 1.97 , indicating that the global root distribution is almost isotropic ( $C O=2$ for isotropic conditions). The model was validated on the basis of independent data from the second experiment. This model enables $R L D$ mapping and description of $R L D$ variability on sorghum from RID observations on trench profiles (with $10 \times 10 \mathrm{~cm}$ or $5 \times 5$ $\mathrm{cm}$ surface area used for RID measurement).
\end{abstract}

Keywords: root architecture, root modeling, root orientation, sorghum (Sorghum bicolor), trenchprofile method.

\begin{abstract}
Abbreviations: $A N$, root anisotropy factor; $C O$, geometric coefficient; RID, root intersection density $\left(\right.$ per $\left.\mathrm{m}^{2}\right) ; R L D$, root length density $\left(\mathrm{m} \mathrm{m}^{-3}\right) ; P$, index of preferential root direction
\end{abstract}

\section{Introduction}

Sorghum (Sorghum bicolor L. Moench) is one of the five major cereals in the world (Krieg 1983, FAO 1996). It is generally considered to be more drought tolerant than maize and is often cropped under rainfall conditions that are too risky for maize (Rai et al. 1999, Baldy et al. 1993, Berenger and Faci 2001). In Soudano-Sahelian areas of West Africa, it is the second most common crop, after millet, and is mostly cultivated between 600 and $800 \mathrm{~mm}$ isohyets (Chantereau and Nicou 1991). Its capability to withstand temporary water shortages is considered to be mainly due to its root system, which can extend into the deep soil layers (Masi and Maranville 1998). It is therefore essential to be able to characterize sorghum root system features, especially the spatial distribution of the root length density $(R L D)$ for the purposes of, for instance, agronomic analyses and variety studies in breeding programs. The $R L D$ spatial distribution, which is linked to the root architecture, determines water and nutrient uptakes (Gregory 2006, Lynch 1995). However, it is hard to assess the $R L D$ spatial distribution in the field due to the variability in root distributions and soil properties and the difficulty to collect sufficient soil and root samples. Several $R L D$ measurement methods have been described (Box 1996), but their implementation in the field is often costly and generates data that are not very representative of the actual root distribution variability.

Mapping root intersections in a soil profile by the trench profile method (Böhm 1976) has the advantage of being feasible in the field while facilitating the study of root distributions in soil (Tardieu 1988,

Chopart JL, Sine B, Dao A, Muller B 2008. Root orientation of four sorghum cultivars: application to estimate root length density from root counts in soil profiles. Plant Root 2: 67-75. doi:10.3117/plantroot.2.67

Copyrights 2008, Plant Root (JSRR), www.plantroot.org 
Vepraskas and Hoyt 1988), but it provides no direct information on $R L D$. Direct empirical relationships between the root intersection density (RID) and RLD have been tested for wheat (Drew and Saker 1980) and maize (Chopart and Siband 1999), but the relationships obtained with this approach were not very robust. A mathematical relationship between the intersection density on the side of a soil cube and the $R L D$ inside the same cube was formulated (Lang and Melhuish 1970, Melhuish and Lang 1968, van Noordwijk 1987) and found to be dependent on the degree of root anisotropy and orientation. The relationship between $R I D$ and $R L D$ was studied on simulated maize root systems, and the results highlighted the complexity and need to take root anisotropy and orientations into account (Grabarnick et al. 1998). The model proposed by Melhuish and Lang (1968) and van Noordwijk (1987) was used and adapted in the field for maize in Côte d'Ivoire and tested in Burkina Faso, leading to the development of a model to describe $R L D$ for maize from root counts in soil profiles in the field (Chopart and Siband 1999). This model was also subsequently tested in Colombia (Chopart et al. 2001, Roveda-Hoyos et al. 2001). It has been used in several field studies, including a recent one in France (Chopart et al. 2007). Approaches based on the studies of Melhuish and Lang (1968) and van Noordwijk (1987), including a measurement method very close to that of Chopart and Siband (1999), were used in root isotropy studies to measure $R L D$ on the basis of RID in tree roots (Lopez-Zamora et al. 2002). This type of research study has never been undertaken on sorghum. Early studies (Blum et al. 1977 a, 1977b) did shed some light on sorghum root systems, but they were mainly descriptive and the relevance of the findings was partially questioned by the results of more recent studies on modern cultivars. Root diameters differ markedly in the sorghum root system, as it is the case for many crops. In order to determine the main root directions, it is important to make a distinction between different root categories because they may have different directions, as already shown in maize (Cahn et al. 1989) and sugarcane (Chopart et al. 2008). However, roots cannot be distinguished in this way if the trench-profile method is used in the field. For our findings to be usable in the field, we thus simply distributed roots in two categories and assumed that thick roots $(>1 \mathrm{~mm}$ diameter) were shoot-borne and those under $1 \mathrm{~mm}$ were lateral branching roots. Preliminary studies based on full root systems have shown that this is actually the case (Chopart, personnal communication)

The present study was conducted with the aim of highlighting the preferential orientations of thick and fine roots in order to develop a robust and cost-effective method for estimating $R L D$ from root intersection counts in soil profiles. These results should be helpful for future studies related to sorghum root growth and architecture.

\section{Materials and Methods}

Experimental design for root anisotropy and direction studies and model development (MD)

An experiment was carried out at Bambey (Senegal) (lat. $14^{\circ} 42^{\prime} \mathrm{N}$, long. $16^{\circ} 28^{\prime} \mathrm{W}$, elevation $17 \mathrm{~m}$ ) during the hot rainy season in 2006 on a sandy soil, which is prevalent in northern areas of Senegal. This area is under a Soudano-Sahelian climate, with a mean annual rainfall of $450 \mathrm{~mm}$, falling from June to October. Four sorghum cultivars (SSM1611, IS16101, SSM249 and SARIASO 10) (Table 1) were sown in July 2006, with $0.8 \mathrm{~m}$ spacing between rows and $0.4 \mathrm{~m}$ spacing between plants in the same row. The experiment was statistically designed as random complete blocks with 4 replications (16 plots). The crop was managed according to the locally recommended cropping method, with tillage and chemical fertilization. Complementary irrigations were applied when necessary to avoid water stress. The mean daily temperatures were never lower than $28^{\circ} \mathrm{C}$.

Table 1. Main features of the cultivars studied for model development (4 cultivars) and model validation ( 2 cultivars)

\begin{tabular}{lcccccccc}
\hline & \multicolumn{3}{c}{ Model development cultivars } & & \multicolumn{2}{c}{ Model validation cultivars } \\
\cline { 2 - 3 } & IS16101 & SSM249 & SSM1611 & SARIASO 10 & & \multirow{2}{*}{ F2-20 } & IRAT 204 \\
\hline Botanic type & Durra & Guinea & Durra & Caudatum & & Caudatum & Caudatum \\
Geographical origin & Cameroon & Burkina Faso & Chad & Burkina Faso & & Senegal & Senegal \\
Sowing-maturity length (days) & 145 & 105 & 145 & 100 & & 110 & 90 \\
Maximum height (cm) & 300 & 250 & 280 & 170 & & 180 & 130 \\
1000 grain weight (g) & 50 & 29 & 55 & 24 & & 25 & 28 \\
\hline
\end{tabular}

\footnotetext{
${ }^{\#}$ F2-20 is also named $87-62$.
} 
Root measurements were obtained in triplicate (three replications per cultivar) at three dates, i.e. at 22, 43 and 103 days after sowing (DAS) for SARIASO 10 and SSM 249 , corresponding to $20 \%, 42 \%$ and $100 \%$ of the cycle, and at 22, 43 and 134 DAS for SSM1611 and IS16101, corresponding to $15 \%, 30 \%$ and $92 \%$ of the cycle (Table 1). Roots were sampled from trench profiles at 0.1 and $0.3 \mathrm{~m}$ from the row (in the inter-row), and at different depths according to root front growth: at 0.1 and $0.3 \mathrm{~m}$ for the first date, at 0.1 , 0.3 and $0.5 \mathrm{~m}$ for the second date, and at $0.1,0.3,0.5$, 0.7 and $1.0 \mathrm{~m}$ for the last date. The standard RID and $R L D$ sampling procedure is based on the extraction of $1 \times 10^{-3} \mathrm{~m}^{3}$ undisturbed soil cubes from open soil profiles using a three-sided partial steel cube sampling device with sharpened edges $(0.1 \mathrm{~m})$ to facilitate soil penetration (Fig. 1a). The sampling device is cautiously pressed through the soil profile until its back edge is level with the soil surface (Fig. 1b). It is then pulled back out of the soil and excess soil from the three open sides of the device is shaved to obtain a soil cube. A second sample is taken at the same depth with the open sides of the sampling device oriented in the opposite direction relative to the first sample in order to get - with the two soil cube samples - six open cube sides for the root counts. Immediately after sampling, roots intersecting the three open sides of the soil cubes are counted. The soil cube sides could be sprayed with water in order to achieve better root intersection visibility. Root intersection counts enable assessment of root intersection densities (RID) for the three open sides (horizontal, vertical-transversal, vertical-longitudinal) of each soil cube, resulting in $3 R I D$, which were expressed on the basis of the cube side surface. Thereafter, the soil cubes are brought to the laboratory and the roots are separated from the soil under running tap water on a sieve (1 mm mesh). In the present study, we counted root intersections, while making a distinction between fine roots (diameter $d<$ $1 \mathrm{~mm})$ and thick roots $(d>1 \mathrm{~mm})$. In the following text we will refer to thick, fine or all roots using ${ }_{t}$, or $_{\mathrm{a}}$ indices, respectively. Then, for each sample, fine root
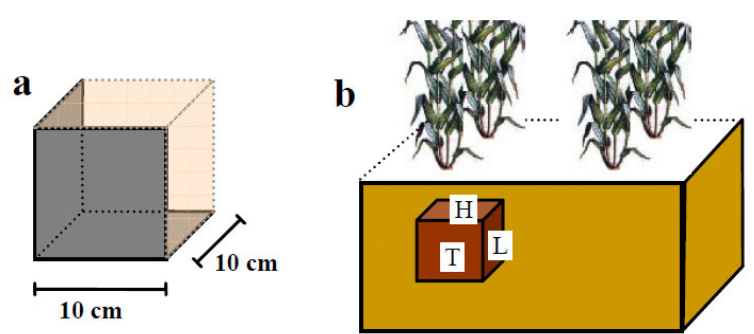

Fig. 1. Root intersection density sampling and measurement procedure. a: a three-sided $\left(0.01 \mathrm{~m}^{2}\right)$ partial steel cube sampling device, $\mathrm{b}$ : a sampling device in the soil profile, with sides oriented according to the soil surface and plant row $(\mathrm{H}$ : horizontal, L: longitudinal, T: transversal). lengths and thick root lengths were determined by the intersection method (Newman 1966, Tennant 1975) in order to obtain the root length density $(R L D)$. A total of 195 samples of $2 \times 10^{-3} \mathrm{~m}^{3}$ of soil each were obtained (three dates, four cultivars, three replications, two distances from the row, and two to five soil depths). Replicate measurements were averaged (same cultivar, same date and same position), resulting in 65 data sets (one sample did not contain any roots).

\section{Experimental design for model validation (MV)}

In order to validate the results and models resulting from the first study, a second experiment was carried out in 2008 at Thiès, Senegal (lat. $14^{\circ} 45^{\prime} \mathrm{N}$, long. $16^{\circ} 53^{\prime} \mathrm{W}$, elevation $70 \mathrm{~m}$ ) during the dry season (colder and drier climatic conditions than MD) on a sandy loam soil with two other cultivars : IRAT 204 and F2-20, also named 87-62 (Table 1). Crop management was similar to that of the first experiment. Irrigations were regularly applied throughout the cycle. Root samples were taken at 57 DAS (corresponding to $63 \%$ of the IRAT 204 cycle and $52 \%$ of the F2-20 cycle) from one pit for IRAT 204 and two pits for F2-20. Soil samples were taken using $0.1 \mathrm{~m}$ diameter and $0.1 \mathrm{~m}$ long metallic cylinders. The samplings were performed at three distances from the rows $(0.1,0.2$ and $0.3 \mathrm{~m})$ and at three depths $(0.1,0.3$ and $0.5 \mathrm{~m})$. Roots were counted on the two open sides of the cylinders, distinguishing fine $(d<1 \mathrm{~mm})$ and thick $(d$ $>1 \mathrm{~mm}$ ) roots, and for each cylinder the two counts were averaged for each root category. $R L D$ were assessed according to the same procedure as in the MD study. Direct triplicate measurements were analyzed, with 27 samples collected, but only 25 were finally considered since two samples did not contain any roots.

\section{Data analysis}

The study involved determining the best method for correlating and ultimately predicting the root length density $(R L D)$ on the basis of root intersection counts in a soil plane. The first approach used to estimate this relationship was to establish simple empirical regressions between root intersection densities (RID) within a monitored unit area and $R L D$. In maize (Chopart and Siband 1999) and sugarcane (Chopart et al. 2008), it was found to be more suitable to study the main root orientations and account for them in the model. Taking this geometrical distribution associated with the root system architecture into account enhanced prediction of the relationship between $R I D$ and $R L D$. The theoretical aspects of the relationship between RID and RLD, while taking the root orienta- 
tion relative to the counting plane into account, were analyzed by van Nordwijk (1987). Measurement of root intersections on three perpendicular sides $(\mathrm{x}, \mathrm{y}, \mathrm{z})$ of a soil cube enables determination of the $A N$ root anisotropy factor (Lang and Melhuish 1970) through the following relationship:

$$
A N=\sqrt{\left(\left(R I D_{x}-R I D_{m}\right)^{2}+\left(R I D_{y}-R I D_{m}\right)^{2}+\left(R I D_{z}-R I D_{m}\right)^{2}\right) /\left(6 R I D_{m}^{2}\right)}
$$

Where $R I D_{\mathrm{m}}$ is the mean $R I D$ of the three sides.

$A N$, dimensionless, ranges from 0 (isotropy) to 1 (parallel roots). If all roots are completely perpendicular to the counting plane, each root intersection would correspond to a root length equal to that of the side of the cube, but no roots can be counted if they are all parallel to the counting plane. According to Melhuish and Lang (1968) and van Noordwijk (1987), $R L D$ can be calculated $(R L D c)$ on the basis of RID measured in a single soil plane $\left(_{\mathrm{x}}\right)$, by taking the root distribution (anisotropy and preferentially orientation) into account. A $P_{x}$ coefficient is calculated for this plane, with ${ }_{y}$ and ${ }_{z}$ representing the other two perpendicular planes.

$P_{x}=\left(R I D_{\mathrm{y}}+R I D_{z}\right) / 2 R I D_{x}$

If $P_{x}>1$ or $<1$, the roots mainly have a parallel or perpendicular orientation relative to the measurement plane $\mathrm{x}$, respectively. According to Melhuish and Lang (1968) and van Noordwijk (1987), the aggregate functions form a single continuous curve relating calculated $R L D c$ to $R I D_{x}$ for all $P_{x}$ values:

$$
\begin{aligned}
& \text { For } P_{x}>1 \\
& \quad R L D c=R I D_{x}\left(16 P_{x}{ }^{2}+8 P_{x}+6\right) /\left(10 P_{x}+5\right)
\end{aligned}
$$

$$
\begin{aligned}
& \text { For } P_{x}<1 \\
& \quad R L D c=R I D_{x}\left(3 P_{x}^{2}+2 P_{x}+1\right) /\left(2 P_{x}+1\right)
\end{aligned}
$$

For $P_{x}=1$ (isotropic distribution)

$$
R L D c=2 R I D_{x}
$$

These equations are used hereafter to calculate $R L D c$ values from $P_{x}$ and $R I D_{x}$. They can be combined in a general equation with a $C O$ coefficient representing the various aggregated factors for $R I D$ in (Eq. 3):

$$
R L D c=R I D_{x} \times C O_{x}
$$

$R L D$ was modeled only on the basis of measurements of root intersections on a vertical perpendicular plane within a sorghum row because this method is most commonly used for studying roots in a soil profile. The reference plane $\left({ }_{x}\right)$ was therefore systematically the vertical-transversal plane. $R L D$ was correlated with RID. Relationships between $R L D$ values measured in the soil cubes $(R L D m)$ and assessed by various models $(R L D c)$ were evaluated taking the slope, standard error of the slope $(S E)$, intercept and regression $\left(R^{2}\right)$ into account. All the regressions obtained were highly significant at $p=$ 0.001 . Differences between measured and calculated $R L D$ were also analyzed by the following statistical quantities: Nash efficiency coefficient $(N E)$ (Nash and Sutcliffe 1970), root mean square error (RMSE) (Loague and Green 1991) and mean bias. NE, RMSE and $M B$ should be as close as possible to $1,0 \%$ and $0 \%$, respectively. $N E$ may have a negative value.

\section{Results}

Main features of the studied cultivar roots and shoots

Only the main features of the studied cultivars were presented here (see also Table 1). A more indepth description and analysis of their development will be presented in another paper. Root depths were similar for the four cultivars at the two first observation dates (22 and $43 D A S$ ), equal to $0.3 \mathrm{~m}$ and $1.4 \mathrm{~m}$, respectively. At $103 D A S$, roots reached $1.8 \mathrm{~m}$ for SSM249 and SARIASO 10, which were at maturity. At 134 $D A S$, the root depth was $2 \mathrm{~m}$ for SSM1611 and IS16101, which were at the grain maturing stage. At $22 D A S$, shoot/root biomass ratios were 8.8, 6.1, 7.1 and 6.9 for SSM249, SARIASO 10, SSM1611 and IS16101, respectively. At $43 D A S$, these ratios were $3.4,9.9$ and 10 for SARIASO 10, SSM1611 and IS16101, respectively. At $103 D A S$, they were found to be 2.9 and 2.4 for SSM249 and SARIASO 10, and at $134 D A S$ they were 3.8 and 2.7 for SSM1611 and IS16101. The number of fine roots was globally tenfold higher than that of thick roots.

\section{Root anisotropy and orientations}

A preliminary analysis in which the results obtained at the three sampling dates were compared showed that there was no change in the extent of anisotropy $(A N)$ or in the root orientation coefficient $P$, for fine and thick roots, over the time course of the study (data not shown). The data obtained at the three measurement dates were thus studied together. Root anisotropy and orientation were characterized by the $A N$ and $P$ index (Eq. 1 and 2) by measuring root intersections on the three perpendicular sides of the soil cubes. Anisotropy levels appeared to be quite similar for the four cultivars (Fig. 2) despite a high range of RID values (Fig. 3) and differences between cultivar features. We thus decided to average data for the four cultivars to assess the overall results (Fig. 4) and to present them in the same figures. 

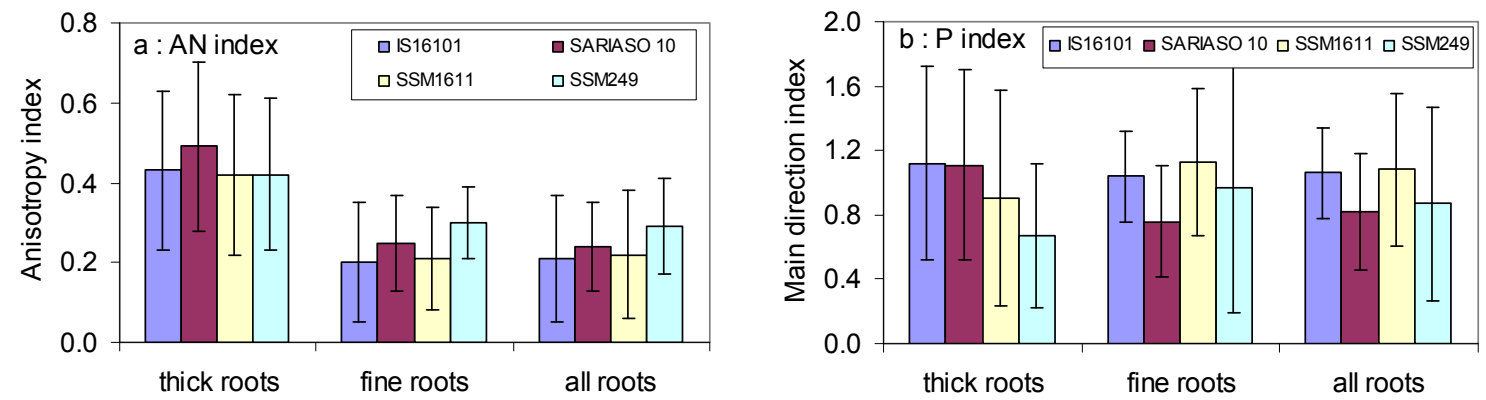

Fig. 2. Comparisons of the average anisotropy index (a) and the average main direction index (b) of the four sorghum cultivars (average of all data for a given cultivar). Standard deviations are shown. $A N$ and $P$ are dimensionless.
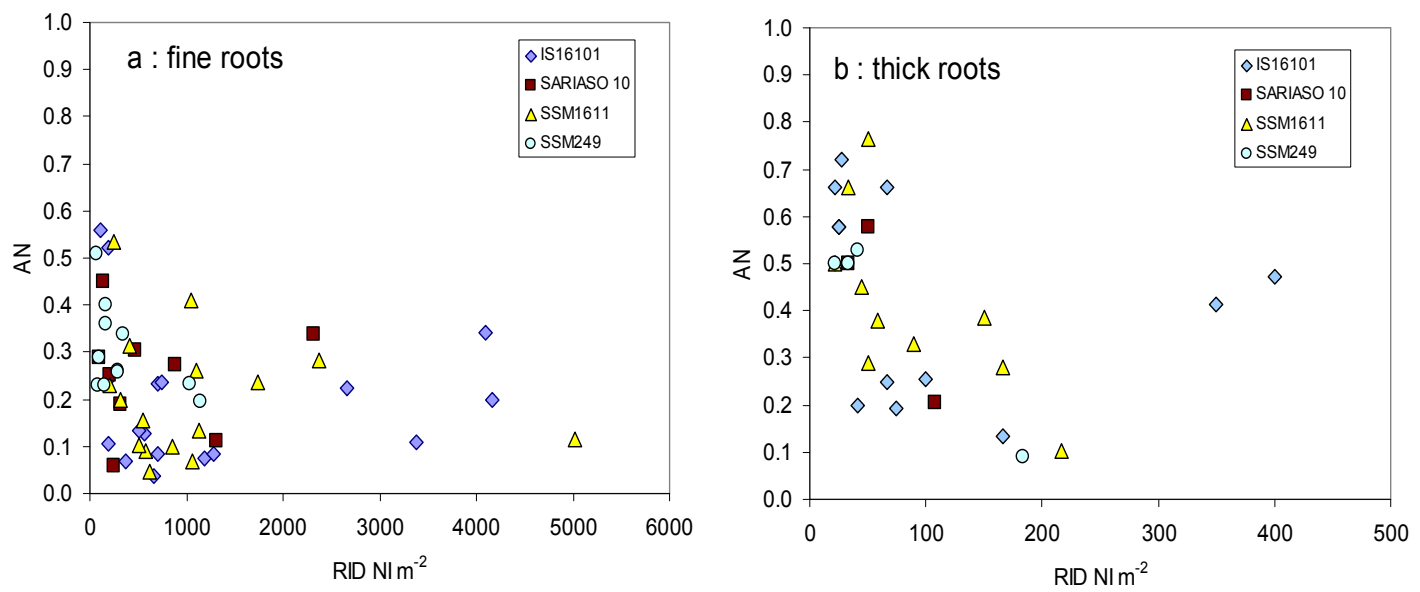

Fig. 3. Variations in the anisotropy index $(A N)$ for fine roots (a) and thick roots (b) according to the root intersection density $(R I D) . A N$ is dimensionless (between 0 and 1 ).

\section{Fine roots}

Considering all the data $(n=53)$, the anisotropy index $(A N)$ was always less than 0.60 (Fig. 3), and the general mean was 0.23 (Fig. 2 and 3), which indicates that the root distribution was close to isotropy. Moreover, the anisotropy index $(A N)$ did not seem to be influenced by the root position in the soil. For all cultivars, there seemed to be a slight preferential root orientation $\left(P_{f}>1\right)$ when the root intersection densities were very low at the deepest horizons (Fig. 4). However, the general mean for $P_{f}$, considering all depths, was 1.0.

\section{Thick roots}

We collected only 29 samples containing at least one thick root count per cube side. The anisotropy indexes $(A N)$ were higher for thick roots than for fine ones (Fig. 2), particularly when the root density was low, i.e. early in the cycle or in the deepest horizons (Fig. 4). Considering all the data, we did not detect any factor that could explain the $P_{t}$ variation, except the crop age (Fig. 4 and 5). At the beginning, $P_{t}$ was low,

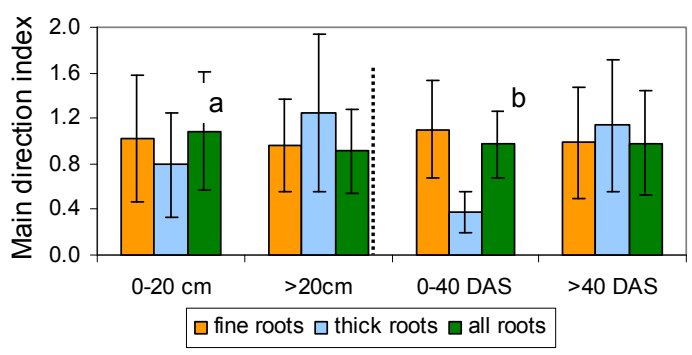

Fig. 4. Variations in the main direction index $(P)$ according to depth (a) and crop age $(D A S)(b)$. Presented $P$ values are averages for the four cultivars. Standard deviations are shown. $\mathrm{P}$ is dimensionless.

close to 0.5 , indicating that thick roots were mainly horizontal. Then $P_{t}$ increased up to 1.5 at the middle of the cycle, revealing that thick roots had mainly switched to a vertical orientation. At the end of the cycle, $P_{t}$ was around 1 , indicating a global isotropic situation, but it was actually highly variable. $P_{t}$ also seemed to slightly vary with depth. Average $P_{t}$ values ranged from 0.7 for SSM249, and at this point the thick roots were more horizontal than those of the other cultivar, to 1.1 for IS16101 and SARIASO 10, 


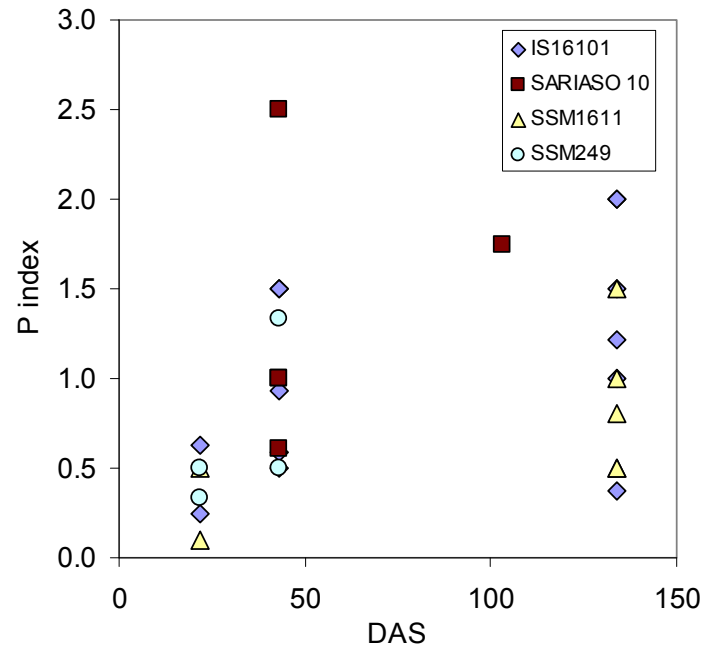

Fig. 5. Variation in the thick root main direction index $\left(P_{t}\right)$ according to the crop age $(D A S) . P$ is dimensionless.

which had more vertical thick roots. Considering all the data, the global $P_{t}$ average was 0.98 , with about 90 thick root intersections per square meter. Due to the limited number of data and their high variability, these should be considered as preliminary results and need to be confirmed.

\section{All roots}

At all dates, for all depths and for the four cultivars, fine roots were much more numerous (about tenfold more) than thick roots. The results obtained when all roots were pooled were thus logically similar to those obtained with fine roots only (Fig. 2 and 4). For each cultivar, the average anisotropy index $(A N)$ was quite low, with a maximum value of 0.29 for SSM249. The $P_{a}$ coefficients were also close to 1 , ranging from 0.87 (for SSM149) to 1.08 (SSM1611), with no significant differences. These roots were therefore globally isotropic. In such conditions, it was not possible to identify any factors able to explain the $P_{a}$ variation.

\section{Prediction of root length density from intersections for all pooled roots}

\section{Geometric model formulation and calibration}

We considered the geometric model presented in equation (4), which includes a coefficient $(C O)$ depending on the index $\left(P_{a}\right)$ of the preferential root direction in relation to the vertical-transversal observation plane. $C O$ must be assessed according to $P_{a}$. However, considering the very low anisotropy of the root distribution, it was not possible to identify any variable that could explain the $P_{a}$ variability, which actually likely depends on several variables. Therefore we decided to use the average $P_{a}$ value for all the data

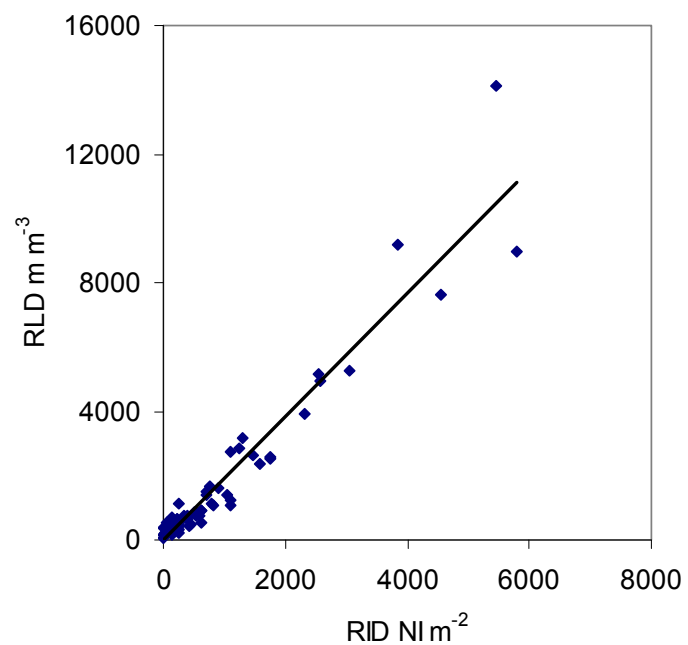

Fig. 6. All pooled roots. Empirical relationship between the observed root intersection density (RID, number of intersections per $\mathrm{m}^{3}$ ) on the transversal side and the measured root length density $\left(R L D m, \mathrm{~m} \mathrm{~m}^{-3}\right)$ in the corresponding cube.

(four cultivars, all dates and depths; $n=56$ ). The mean $P_{a}$ was 0.98 (Fig. 2). Then according to (Eq. 3) the mean $C O_{a}$ was 1.97 . These $C O_{a}$ coefficients were then used to develop models to correlate $R I D$ and $R L D$, while taking the root geometry into account:

$R L D_{a}=1.97 R I D_{a}$ tion

Empirical linear model formulation and calibra-

According to the above presented results (Figs. 2, 3 and 4) which highlight the low anisotropy of sorghum roots, another simpler approach could be used that ignores root orientation variations and establishes a direct relation between the root intersection density (RID) counted on the transversal cube side and the root length density $(R L D)$ measured inside the cube. The linear regression between $N I_{a}$ and $R L D_{a}$ (Fig. 6) is:

$R L D_{a}=1.92 R I D_{a} \quad n=64 \quad R^{2}=0.921$

Equation (6) is the empirical model we proposed. The two models, geometric and empirical, are quite similar. Both allow a good estimation of the measured RLD (Fig. 7).

Empirical model: $R L D c_{a}=0.914 R L D m_{a}+174$

$$
n=64 \quad R^{2}=0.921
$$

Geometric model: $R L D c_{a}=0.938 R L D m_{a}+178$

$$
n=64 \quad R^{2}=0.921
$$


Table 2. Model validation, all pooled roots. Characteristics and analysis of linear regressions between measured and calculated root length densities ( $R L D m$ and $R L D c, \mathrm{~m} \mathrm{~m}^{-3}$ ) with the two tested models, geometrical (Eq. 5) and empirical (Eq. 6)

\begin{tabular}{|c|c|c|c|c|c|c|c|}
\hline Cultivars & Model (no. Eq.) & Slope & Intercept & $R^{2}$ & $M B^{\mathrm{a}}$ & $N E^{\mathrm{b}}$ & $R M S E^{\mathrm{c}}$ \\
\hline \multirow{2}{*}{$\begin{array}{l}\text { IRAT } 204 \\
n=7\end{array}$} & empirical. (4) & 0.98 & +48 & .932 & -1.3 & 0.93 & 19.0 \\
\hline & geometrical (10) & 0.96 & +48 & .932 & -3.4 & 0.92 & 19.4 \\
\hline \multirow{2}{*}{$\begin{array}{l}\mathrm{F} 2-20 \\
n=18\end{array}$} & empirical. (5) & 1.05 & +96 & .900 & +4.4 & 0.89 & 17.2 \\
\hline & geometrical (13) & 1.03 & +96 & .900 & +2.9 & 0.90 & 16.7 \\
\hline \multirow{2}{*}{$\begin{array}{l}\text { IRAT } 204 \text { and F2-20 } \\
n=25\end{array}$} & empirical. (6) & 1.02 & +115 & .911 & +2.8 & 0.91 & 17.7 \\
\hline & geometrical (13) & 1.00 & +115 & .911 & -0.7 & 0.91 & 17.5 \\
\hline
\end{tabular}

${ }^{\mathrm{a}}$ : mean bias (\%), ${ }^{\mathrm{b}}$ : Nash efficiency coefficient, ${ }^{\mathrm{c}}$ : root mean square error $(\%)$.

no. Eq. indicates the number of equation described in the text.

All regressions were significant at $p<0.001$.

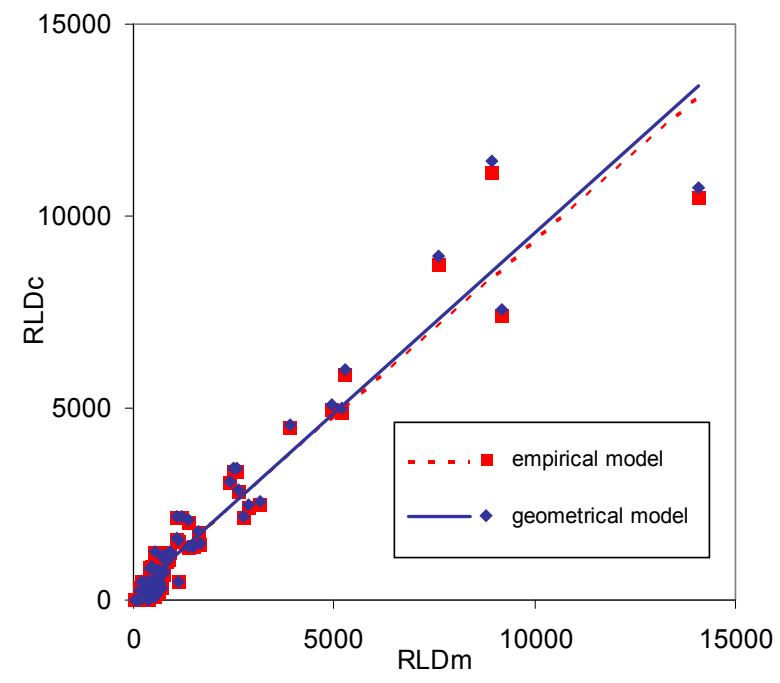

Fig. 7. Model calibration for all pooled roots. Relationship between measured root length density $\left(R L D m, \mathrm{~m} \mathrm{~m}^{-3}\right)$ and calculated root length density $\left(R L D c, \mathrm{~m} \mathrm{~m}^{-3}\right)$ using geometric (5) and empirical (6) models.

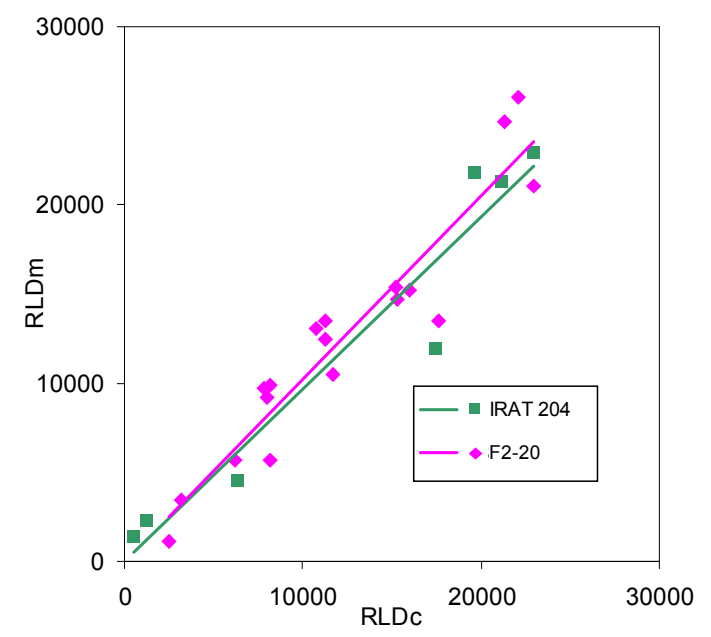

Fig. 8. Model validation for all pooled roots. Relationship between measured $\left(R L D m, \mathrm{~m} \mathrm{~m}^{-3}\right)$ and estimated root length densities using the proposed geometric model $(R L D c=R I D$ $\times C O)$. Regression features are given in Table 2 .

\section{Model test}

The empirical (Eq. 6) and geometric (Eq. 5) models developed for estimating $R L D$ from $R I D$ were tested with an independent dataset derived from the model validation (MV) experiment (different site, two different varieties, different year). The validation results are summarized in Table 2 and Fig. 8 (Fig. 8 presents only the results relative to the geometrical model since both models are very similar). The empirical linear and geometric models were found to have equivalent and satisfactory prediction capacities, without marked bias (Table 2, Fig. 8), for both cultivars, despite the fact that they were quite different (Table 1). It is therefore possible to just use one of these models and we suggest using the geometrical model since it is slightly more accurate (Table 2 ) and mechanistic.

\section{Discussion and Conclusion}

This study generated new information on sorghum root orientations in the soil, and models correlating $R L D$ and $R I D$ were developed and validated on the basis of these findings.

\section{Root orientation}

In the present study, thick roots $(d>1 \mathrm{~mm})$ extending from the shoot (nodal roots) had an horizontal orientation during the first stage of cycle and (or) close to the surface (Fig. 4), and a vertical orientation in deeper soil layers (from $0.2 \mathrm{~m}$ depth to the root front) where fewer roots were present. Fine roots $(d<1 \mathrm{~mm})$ branching from the nodal roots appeared to be relatively isotropic (Figs. 2, 3, 4). Quantitative results obtained on the main orientations of two root types, i.e. oriented thick roots and isotropic fine roots, provide insight into the architecture of sorghum roots, which is still only partially understood. 
Modeling $R L D_{a}$ from $R I D_{a}$ : relevance and advantages of the method

According to a theoretical model (Lang and Melhuish 1970, van Noordwijk 1987), the relationship between $R I D$ (counted on the sides of a cube) and RLD depends on the root orientation relative to the counting side. The geometric model proposed requires calibration with RID observed on three soil planes but could be used for $R L D$ prediction in the field on the basis of RID measured in a single vertical plane. For all roots, the $P_{a}$ index (Eq. 2) was around 1 (Fig. 2), revealing an almost isotropic distribution. The orientation coefficient $(\mathrm{CO})$, mechanistically based on the root distribution geometry (Eq. 3 and 4), could be considered as stable $\left(C O_{a}=1.97\right)$, independently of the cultivar, age and depth, whereas van Noordwijk's model proposes a $C O$ of 2 for isotropic distributions. We successfully validated the model for two other cultivars with quite different morphologies (Table 1), cropped at a different site and under different climatic conditions. Six cultivars with different genetic features were thus considered when designing and testing the model. However, for both phases, the cropping conditions were quite similar and suitable for growth, i.e. tropical or subtropical climate, fertilizer applications, deep soil without stones or excessive water. This indicates that the model is robust and can be used for other tropical or sub-tropical varieties under similar conditions. However, nothing could be affirmed about the possibility of using it under very different climatic or soil conditions without further testing.

The proposed model, which assesses all roots, is quite simple and also very close to the theoretical model (Melhuish and Lang 1968, Lang and Melhuish 1970). It was not necessary to consider a second experimental and $R I D$-dependant coefficient as with maize (Chopart and Siband 1999) or sugarcane (Chopart et al. 2008), nor to establish a non-linear relationship between NID and $R L D$ (Lopez-Zamora et al. 2002).

The results were accurate enough for $R L D$ estimates in the field, when the user requires easy data acquisition for the analysis of root distribution in a soil. For studies on soil-crop relationships or water and nutrient uptake, it is now useful to map root intersections on a plane (trench profile) or in rhizotrons in order to determine both the root length density $(R L D)$ and its spatial variability, which are standard root parameters for water and nutrient uptake. The method for running this model is simple and involves opening a pit and counting root intersections in the profile by mapping them on a $10 \times 10 \mathrm{~cm}$ or $5 \times 5 \mathrm{~cm}$ grid, thus taking spatial variability in the root distribution into account. Complicated standard procedures (sampling, root extraction, preparation and length measurement) are thus not required unless obtaining highly accurate results is a prime concern.

\section{Acknowledgments}

This study was carried out in CERAAS, within ARPEGE CIRAD project. The authors sincerely thank Amadou Diop and Sidi Sidibe for their valuable help during the agronomical trials. The second author is very grateful to Dr. Serge Braconnier (CIRADCERAAS) and Dr. Aliou Guissé (University of Dakar) for providing him with a doctoral fellowship and for their assistance. The first author acknowledges CERAAS for inviting him to participate in the research.

\section{References}

Baldy C, Konaté JM, Olufayo A, Ruelle P, Fernandes A 1993 Résistance à la sécheresse du sorgho grain en climat méditerranéen et gestion optimale d'une quantité d'eau limitée. Sécheresse. 4(2): 85-93. (in French with English abstract)

Berenguer MJ, Faci JM 2001 Sorghum (Sorghum Bicolor L. Moench) yield compensation processes under different plant densities and variable water supply. Europ. J. Agron. 15: $43-55$.

Blum A, Arkin GF, Jordan WR 1977a Sorghum root morphogenesis and growth. I. Effect of maturity genes. Crop Sci. 17:149-153.

Blum A, Arkin GF, Jordan WR 1977b Sorghum root morphogenesis and growth. II. Manifestation of heterosis. Crop Sci. 17:153-157.

Böhm W 1976 In situ estimation of root length at (natural soil profiles. J. Agric. Camb. 87:365-368.

Box JE 1996 Modern methods for root investigations. In: Waisel Y, Eshel A, Kafkafi U, Eds., Plant roots the hidden half. Marcel Dekker, New York, USA, pp 193-237.

Cahn, MD, Zobel RW, Bouldin DR 1989 Relationship between root elongation rate and diameter and duration of growth of lateral roots of maize. Plant Soil 119:271-279.

Chantereau J, Nicou R 1991 Le Sorgho. Maisonneuve \& Larose, Paris, pp. 1-159. (in French).

Chopart JL, Siband P 1999 Development and validation of a model to describe root length density of maize from root counts on soil profiles. Plant Soil 214:61-74.

Chopart JL, Siband P, Dingkhun M, Roveda G, Baquero J 2001 From single plane profiles to root length density in space. Validation of a model in the field. Root Res. 10(Extra issue):586-567.

Chopart JL, Payet N, Saint Macary, Vauclin M 2007 Is maize root growth affected by pig slurry application on a tropical acid soil? Plant Root 1:75-84.

Chopart JL, Rodrigues SR, de Azevedo MC, de Conti Medina C 2008 Estimating root length density through root mapping and orientation modelling Plant Soil DOI:10.1007/s11104-008-9683-4.

Drew MC, Saker LR 1980 Assessment of a rapid method using soil cores for estimating the amount and distribution of crop roots in the field. Plant Soil 55:297-295.

FAO 1996 The world sorghum and millet economies. Facts, trends and outlook. FAO, Rome, pp. 1-68. 
Grabarnik P, Pages L, Bengough A 1998 Geometrical properties of simulated maize root systems: consequences for length density and intersection density. Plant Soil 200:157-167.

Gregory PJ 2006 Plant roots: Growth, activity and interactions with soils. Blackwell Publishing Ltd, Oxford, UK, pp. $1-318$.

Krieg DR 1983 Sorghum. In: Teare ID, Peet MM, Eds., Crop water relations. John Wiley and Sons, New York, pp 351-388.

Lang ARG, Melhuish FM 1970 Length and diameters of plant roots in non-random populations by analysis of plane surface. Biometrics 26:421-431.

Loague K, Green RE 1991 Statistical and graphical methods for evaluating solute transport models: Overview and applications. J. Contam. Hydrol. 7:51-73.

Lopez-Zamora I, Newton F, Comerford NB, Barros NF 2002 Root isotropy and an evaluation of a method for measuring root distribution in soil trenches. For. Ecol. Manage. 60:303-310.

Lynch J 1995 Root architecture and plant productivity. Plant Physiol. 109:7-13.

Masi CEA, Maranville JW 1998 Evaluation of sorghum root branching using fractals. J. Agric. Camb. 131: 259-265.

Melhuish FM, Lang ARG 1968 Quantitative of root in soil. I Length and diameters of cotton roots in a clay-loam soil by analysis of surface-ground block of resin-impregrated soil. Soil Sci. 106:16-22.

Nash JE, Sutcliffe JV 1970 River flow forecasting through conceptual models. J. Hydrol. 10:282-290.

Newman EI 1966 A method of estimating the total length of roots in a sample. J. App. Ecol. 3:139-145.

Rai NK, Murty DS, Andrews DJ, Bramel-Cox PJ 1999 Genetic enhancement of pearl millet and sorghum for the semi-arid tropics of Asia and Africa. Genome 42: 617-628.

Roveda-Hoyos G, Chopart JL, Baquero JE, Rojas LA, Dingkuhn M 2001 Modeling growth and distribution of maize (Zea mays L.) roots under field conditions in the eastern plains of Colombia. In: Horst WJ, Schenk MK, Bürkert A, Claasen N, Flessa H, Frommer WB, Goldbach H, Olfs HW, Römheld V, Sattelmacher U, Schubert S, von Wirén N, Wittenmayer L, Eds., Plant nutrition - Food security and sustainability of agro-systems. Kluwer Academic Publishers, The Netherlands, pp. 576-577.

Tardieu F 1988 Analysis of the spatial variability of maize root density. I. Effect of wheel compaction on the spatial arrangement of roots. Plant Soil 107:259-266.

Tennant D 1975 A test of a modified line intersect method of estimating root length. J. Ecol. 63:955-1001.

van Noordwijk M 1987 Method for quantification of root distribution patterns and root dynamics in the field. In: International Potash Institute, Ed., 20th Symposium. Bern, Switzerland, pp. 247-256.

Vepraskas MJ, Hoyt GD 1988 Comparison of the trench-profile and core methods for evaluating root distribution in tillage studies. Agron. J. 80:166-172.

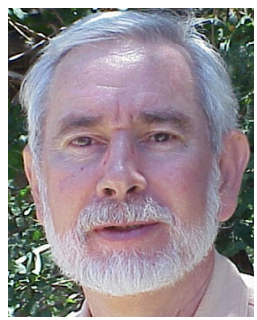

Dr. Jean-Louis Chopart is an agronomist and soil physicist, with special research interest in the root system as water uptake organ and as an indicator of soil physics. $\mathrm{He}$ is working on sugarcane sustainable water management and irrigation.

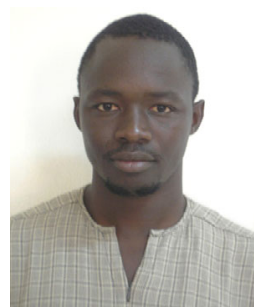

Bassirou Sine is a Senegalese agrophysiologist. His $\mathrm{PhD}$ deals with selection for sorghum drought adaptation. He studied different sorghum traits and particularly the contribution of root architecture to water stress resistance.

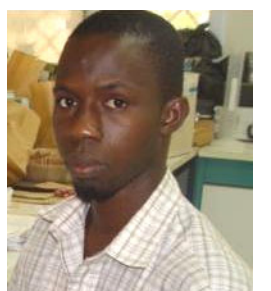

Abdalla Dao is a Burkinabe agrophysiologist student willing at being a plant breeder. He carried out his Master study on sorghum root architecture and development, and he is currently working on water stress resistance of maize.

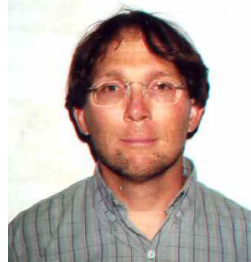

Dr. Bertrand Muller is agronomist and agrometeorologist, with experience on crop modelling. $\mathrm{He}$ is working on improvement and use of crop models for yield forecasting, crop monitoring and development of farmers insurance tools in Soudano-sahelian areas. 